\title{
Diacronie
}

Studi di Storia Contemporanea

$N^{\circ} 17,1 \mid 2014$

Periferie. Cultura, economia, politica

\section{L'Islam di Stato. La figura di Necmettin Erbakan nella Turchia contemporanea}

\section{Emanuela Locci}

\section{(2) OpenEdition}

\section{Journals}

\section{Edizione digitale}

URL: http://journals.openedition.org/diacronie/1100

DOI: 10.4000/diacronie. 1100

ISSN: 2038-0925

\section{Editore}

Association culturelle Diacronie

Notizia bibliografica digitale

Emanuela Locci, «L'Islam di Stato. La figura di Necmettin Erbakan nella Turchia contemporanea », Diacronie [Online], $\mathrm{N}^{\circ}$ 17, 1 | 2014, documento 10, Messo online il 01 mars 2014, consultato il 19 avril 2019. URL : http://journals.openedition.org/diacronie/1100; DOI : 10.4000/diacronie.1100 


\section{Diacronie}

10/

\section{L'Islam di Stato. La figura di Necmettin Erbakan nella Turchia contemporanea}

Emanuela LOCCI*

Fin dai suoi albori la repubblica turca ideata da Atatürk ha rifiutato l'islam politico, facendo di questa negazione uno dei suoi cardini. Necmettin Erbakan, scaltro e navigato uomo politico, ha cercato per anni di scardinare questo principio e ha rappresentato la frangia più importante dell'Islam politico in Turchia. La sua parabola è stata una cartina di tornasole degli avvenimenti politici e sociali che hanno interessato il paese, a partire dagli anni Settanta del secolo scorso fino alle soglie del Duemila. La sua statura politica lo ha reso un attore della scena politica turca e uno degli uomini più temuti dal sistema militare, che in Turchia ricopre da sempre un ruolo di primo piano per la stabilità dello Stato. Scopo di questo saggio è scoprirne la figura e l'opera politica, contestualizzandole all'interno del sistema politico turco.

\section{Una breve biografia}

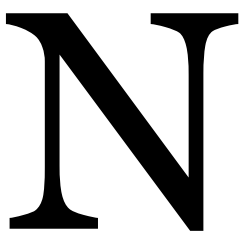

ecmettin Erbakan nacque a Sinope nel 1926: il padre era un giudice della corte penale. Dopo essersi laureato in ingegneria a Istanbul nel $1948^{1}$, proseguì gli studi in Germania per poi stabilirsi nuovamente a Istanbul e insegnare nella stessa università dove si era laureato. La sua carriera politica iniziò alla fine degli anni Sessanta, quando fu eletto deputato tra gli indipendenti ${ }^{2}$. La sua ideologia si delineò più precisamente l'anno successivo, quando

\footnotetext{
${ }^{1}$ Durante gli anni universitari Erbakan conobbe, tra gli altri, Suleyman Demirell in quanto era il capo del suo gruppo di preghiera e membro della confraternita Nașbendiyye.

${ }^{2}$ Necmettin Erbakan, sub vocem, Encyclopaedia Britannica online,
} 
fondò il Partito per l'Ordine Nazionale (Millî Nizam Partisi) che presentava istanze islamiste3. Questo partito era supportato dalla piccola borghesia presente nelle province, che non avevano beneficiato dello sviluppo economico degli anni Sessanta ${ }^{4}$. A questa prima, timida, apparizione seguì lo scioglimento del partito islamico nel $1971 \mathrm{e}$ la rifondazione, due anni dopo, sotto il nome di Partito per la Salvezza Nazionale (Millî Selamet Partisi). Il suo leader divenne vicepremier quando si unì in coalizione con il partito guidato da Ecevit5, il Partito Popolare Repubblicano (Cumhuriyet Halk Partisi), CHP. Nel 1977, Erbakan partecipò al governo guidato da Süleyman Demirel ${ }^{6}$, ma in seguito al colpo di Stato militare del 12 settembre 1980, fu allontanato dalla scena politica e arrestato. Il diritto di esercitare l'attività politica gli fu restituito nel 1987. Fu un ritorno in grande stile, visto che il suo partito era ormai diventato il più importante della Turchia, con una tendenza confermata dalle elezioni di metà anni Novanta. Nel luglio del 1996 divenne primo ministro, alla guida del Partito della Prosperità (Refah Partisi), il primo esponente politico appartenente a un partito islamico a ottenere questa carica dai tempi di Atatürk. Costretto alle dimissioni pochi mesi dopo, a causa delle forti pressioni politiche esercitate dai militari, nel 1998 fu nuovamente allontanato dalla politica per cinque anni. In seguito, Necmettin Erbakan ha fatto parte del Partito della Felicità (Saadet Partisi) l'unico nel panorama politico turco a essere apertamente favorevole al superamento della scelta laicista imposta alla Turchia da

\footnotetext{
URL: < http://www.britannica.com/EBchecked/topic/191078/Necmettin-Erbakan > [consultato il 8 gennaio 2014].

3 ZÜRKER, Erik, Storia della Turchia, Roma, Donzelli, 2007, p. 438.

4 NASSER, Momayezi, «Civil-Military Relations in Turkey», in International Journal on World Peace, 15, 3/1998, p. 15.

5 Bület Ecevit nacque a Istanbul nel 1925, abbandonati gli studi di letteratura fece studi di giornalismo negli Stati Uniti. Tornato in patria nel $1957 \mathrm{fu}$ eletto al Parlamento e da quel momento iniziò la sua lunga carriera politica che lo vide leader del Partito della Sinistra Democratica. Fu primo ministro per cinque mandati. Nel 1974 prese la decisione di invadere Cipro. Negli anni Ottanta fu bandito dalla politica. Si riproporrà anni dopo, nel 1999 vinse le elezioni e rimase a capo del governo fino al 2002, quando il suo partito fu sconfitto alle elezioni. ${ }^{6}$ Süleyman Demirel è nato nel 1924 a Isparta. Laureato in ingegneria, nel 1960 entrò nel mondo degli affari, alle dipendenze di una società statunitense. La sua carriera politica iniziò quattro anni dopo, quando venne eletto leader del Partito della Giustizia. La sua ascesa subì uno stop nel 1980 a causa del colpo di Stato. La sua lunga carriera politica lo vide cinque volte primo ministro (1965-71; 1974-78; 1991-93) e nono Presidente della Repubblica di Turchia dal 1993 al 2000, stesso anno in cui il suo partito scomparve dalla scena politica nazionale.
} 
Atatürk e dai militari. Erbakan morì a Ankara a causa di problemi cardiaci nel 2011, all'età di ottantaquattro anni ${ }^{8}$.

\section{Gli ideali e le linee guida}

Le formazioni politiche a cui Erbakan prese parte erano tutte accomunate da alcuni punti. Il più importante era una visione dell'Islam in senso politico9, affinché non fosse vissuto come una questione personale, secondo quanto ideato da Atatürk, ma come una religione presente nelle scelte quotidiane, anche quelle politiche. Un ritorno a un Islam puro, con le sue regole, restrizioni e ruoli. Un altro punto collegato al primo era la lotta contro l'occidentalizzazione della Turchia. In un periodo, successivamente alla Seconda guerra mondiale, in cui la Turchia si era avvicinata sensibilmente all'Europa e all'Occidente in genere, Erbakan chiedeva un passo indietro, un nuovo nazionalismo. Vi era anche la questione di Israele, che Erbakan considerava alla stregua di un nemico dello stato nazionale. Egli si dichiarava antisionista e antisemita, ma non antigiudaico, descrivendo spesso il giudaismo come una religione meritevole di rispetto ${ }^{10}$. Non secondaria era anche la sua spinta antistatunitense ${ }^{11}$, che rendeva di dominio pubblico organizzando frequentemente manifestazioni di protesta contro la presenza degli Stati Uniti in Turchia ${ }^{12}$. Rimase famosa la sua condanna all'embargo imposto alla Libia di Gheddafi nel 1992, di cui chiese l'abrogazione ${ }^{13}$. Strenuo oppositore dell'ingresso della Turchia nell'Unione Europea, voleva, invece, creare un'Unione Islamica che raccogliesse tutti i paesi musulmani, e con questo obiettivo, nel 1996 e nel 1997,

7 Il Saadet Partisi alle politiche del 2002 ottenne il 2,5\% dei voti, insufficienti a superare la soglia di sbarramento del 10\% prevista nel sistema elettorale turco. Il partito però è l'unico ad avere una struttura organizzativa diffusa sul territorio e spesso svolge più una funzione sociale che politica. Grazie a questo suo radicamento territoriale, SP ha ottenuto alle amministrative del 2004, il 4,1\% dei voti. Il magro risultato del 2002 era stato determinato dall'affermazione del Partito per la Giustizia e lo Sviluppo, anch'esso filo-islamico, anche se più moderato.

8 FAULKNER, Thomas, «Necmettin Erbakan obituary», in The Guardian, 28 February 2001, p. 2.

9 L'espressione "Islam politico" è stata coniata dall'orientalista francese Oliver Roy nel libro ROY, Oliver, L'Échec de l'Islam politique, Paris, Le Seuil, 1992. Non è facile dare una definizione certa del concetto di Islam politico, in generale si rifà alla dottrina portata avanti dai Fratelli Musulmani in Egitto, ma la Turchia presenta delle caratteristiche peculiari soprattutto rispetto alla sua società civile, suddivisa in due blocchi. Da un lato chi ha uno stile di vita occidentale, laico e moderno, dall'altro quanti seguono uno stile di vita improntato ai dogmi religiosi. Il linguaggio politico occidentale fa spesso un uso ampio, elastico e scorretto di questi termini, confondendo l'islamismo con l'Islam.

${ }^{10}$ MUSAWI, Sadrodin, «Erbakan's anti-Zionist Identity», in Islamic Awakening, 1, 8/2013, p. 6.

11 Gli Stati Uniti durante il governo Erbakan del 1996 continuarono a mantenere un atteggiamento di distanza, non lo invitarono a Washington, ma seguirono sempre il protocollo previsto.

${ }_{12}$ MANGO, Andrew, Turkey, the Challenge of a New Role, London, Praeger, 1994, p. 17.

13 MAKOVSKY, Alan, «How to deal with Erbakan», in Middle East Quaterly, 3/1997, p. 4. 
Erbakan visitò vari paesi musulmani per portare avanti il suo progetto, ma le sue dimissioni dal governo, nel 1997, lo costrinsero ad interrompero questo disegno ${ }^{14}$.

\section{Prime esperienze politiche}

Da sempre legato agli ambienti della destra conservatrice turca, venne alla ribalta sulla scena politica nazionale nel 1969, con la nomina a presidente dell'Unione delle Camere di Commercio e di Industria. Durante la sua campagna elettorale aveva cercato di rappresentare gli interessi della piccola e media borghesia industriale, composta dagli uomini d'affari emergenti in quel periodo. Aveva accusato Süleyman Demirel leader di destra del Partito della Giustizia (Adalet Partisi) di avere un atteggiamento servizievole e remissivo nei confronti delle società che possedevano ingenti capitali, specialmente quelle straniere. Da subito nei discorsi di Erbakan si insinuò una nota religiosa, in particolare quando accusò Demirel di aver dimenticato i valori islamici per proteggere gli interessi di sionisti e massoni ${ }^{15}$. Nello stesso 1969 lasciò il partito e si candidò al Parlamento come indipendente riuscendo a ottenere il seggio in rappresentanza della città di Konya ${ }^{16}$.

L'anno successivo, insieme ad altri due deputati indipendenti, fondò il primo partito di ispirazione islamica, il Partito dell'Ordine Nazionale. Per la prima volta nella storia della Repubblica, anche quella parte della società turca che si riconosceva nell'Islam politicizzato ebbe modo di essere rappresentata ${ }^{17}$. L'ideologia portata avanti dal partito entrava in collisione con la dottrina kemalista di uno stato laico, per la quale l'Islam era religione nazionale ma non doveva sovrapporsi alle questioni statali. Dopo pochi mesi di attività il partito fu dichiarato fuorilegge dalla Corte Costituzionale, che ravvisava nei suoi fondamenti la violazione della norma che vietava l'uso della religione per scopi politici ${ }^{18}$. Quando il partito venne dichiarato fuorilegge il suo leader lasciò la Turchia e si trasferì per un breve periodo in Svizzera.

${ }^{14}$ MAZLUMI, Mohammad Reza, «Erbakan's Most Popular Speeches», in Islamic Awakening, 1, 8/2013, p. 8.

${ }^{15}$ Questa posizione si rifà alla tesi del complotto ebraico massonico che investì anche la Turchia nei primi anni Sessanta del Novecento.

${ }^{16}$ La città di Konya era una roccaforte dell'Islam politico.

${ }^{17}$ KRAMER, Heinz, A changing Turkey, Washington, Brookings Institution Press, 200o, p. 64.

18 Ibidem, p. 65. 
Accanto al partito, Erbakan aveva creato un'altra organizzazione conosciuta come Millî Görüş̧(Punto di Vista Nazionale) un'associazione di chiara ispirazione islamica che si occupava di tutti gli aspetti della vita degli aderenti ${ }^{19}$.

Nel 1972 Erbakan e i suoi fedelissimi fondarono un altro partito, denominato Partito della Salvezza Nazionale, che si dichiarava democratico, ma che si richiamava costantemente ai valori fondanti dell'Islam. Con questo partito l'Islam politico si ripropose con fermezza nel panorama politico turco. Erbakan fece della religione il suo cavallo di battaglia, mostrandosi apertamente contrario alla volontà dei governi che si erano susseguiti al comando del paese e che intendevano occidentalizzare la nazione invece di avvicinarsi ai paesi arabi ${ }^{20}$. Alle elezioni del 1973 il partito ottenne l'11,8\% dei voti: era la prima volta che un partito di ispirazione islamica otteneva un tale risultato. Anche se altri partiti, per allargare la propria base elettorale usavano la religione come strumento, Erbakan utilizzava un linguaggio che faceva riferimento ad un Islam nazionalista, ben lontano sia dallo stato laico di Atatürk, sia dall'Islam moderno e razionale voluto dai governanti negli anni Cinquanta. Il partito si sviluppò fino al colpo di Stato del settembre 1980, quando venne dichiarato fuorilegge, come del resto tutti gli altri partiti esistenti. Erbakan subì una condanna a dieci anni di esclusione dalla vita politica attiva.

\section{Le elezioni del 1973, una svolta nazionale}

Le elezioni dell'ottobre 1973 si tennero in un clima teso: la situazione economica del paese era particolarmente delicata, perché all'industrializzazione del paese non era corrisposto un progresso sul piano sociale e l'esercito si sentiva defraudato del suo ruolo di difensore del kemalismo. Regnava un clima di forte instabilità e la politica non riusciva a dare risposte concrete; i partiti attraversavano un periodo di grande insicurezza, caratterizzata da frequenti scissioni e contrasti interni.

Alla tornata elettorale parteciparono otto partiti: tra essi vi era l'MSP, che aveva come presidente Suleyman Arif Emre, ma che vedeva in Necmettin Erbakan il suo uomo di punta. Durante la campagna elettorale furono le dichiarazioni di quest'ultimo a chiarire la posizione del partito: Erbakan si presentava con una chiara ispirazione

\footnotetext{
19 Fino alla fine degli anni Novanta era un movimento che aveva come base geografica la Turchia e non aveva ancora avuto l'occasione di espandersi. Dopo tale data si affermò anche fuori dai confini nazionali, creando sezioni anche nei paesi europei. Oggi l'associazione è presente soprattutto in Germania, ma anche in Francia, Paesi Bassi, Austria, Italia ed in altri paesi europei per un totale di circa mezzo milione di membri.

${ }^{20}$ NOCERA, Lea, La Turchia Contemporanea, Roma, Carocci, 2011, p. 64.
} 
islamica, che intendeva fare propri alcuni principi del socialismo e del capitalismo e utilizzarli in chiave nazionalista. Per le sue dichiarazioni di stampo religioso fu spesso oggetto di attacchi degli organi di stampa, che avanzarono l'ipotesi che il partito ricevesse sovvenzioni dai paesi arabi, in particolare dalla Libia di Gheddafi, che voleva riportare la Turchia nell'orbita dei paesi musulmani ${ }^{21}$. La polizia tenne sotto stretta sorveglianza alcuni esponenti del partito e secondo le autorità vi era la possibilità che il partito stesse portando avanti azioni per cambiare il sistema statale, da laico a confessionale. Il risultato elettorale mise in luce che il caposaldo kemalista della laicità statale, considerato inviolabile, era stato svuotato del suo significato intrinseco, ciò a causa della nascita del partito islamico, sintomo della nascita o rinascita di un islam politico nel paese. Dopo le elezioni ci si aspettava la formazione di un governo di coalizione, che vedeva in prima posizione il CHP di Bület Ecevit e come suo alleato l'MSP di Necmettin Erbakan, lasciando l'AP di Demirel come maggiore partito all'opposizione. Le cose non andarono così perché per questioni interne al partito islamico in un primo momento Erbakan rifiutò l'offerta di Ecevit, aprendo una stagione di forte instabilità politica. Il bandolo della matassa si sciolse alla fine di gennaio del 1974, quando i due partiti si allearono dando vita a un governo di coalizione che vedeva Ecevit primo ministro e Erbakan suo vice ${ }^{22}$. Era la prima volta che un esponente di un partito filoislamico poteva andare al potere, anche se non come primo partito, in un'alleanza che vedeva preponderante il CHP. Il governo rimase in carica per circa nove mesi, durante i quali affrontò la questione di Cipro, la cui risoluzione accrebbe molto la popolarità di Ecevit, che pensò di dimettersi mentre era all'apice della popolarità per poi ripresentarsi da vincitore alle elezioni anticipate già in programma. Le cose non andarono in questo senso e si aprì per la Turchia un quinquennio denso di avvenimenti, contrassegnato dalla costante discontinuità politica ${ }^{23}$.

\footnotetext{
${ }^{21}$ CARRETTO, Giacomo E., «La crisi di governo in Turchia e la formazione del governo Ecevit», in Oriente Moderno 54, 1 3/1974, p. 40.

${ }^{22}$ Gli esponenti del MSP che facevano parte del governo Ecevit erano Süleyman Arif Emre, Ministro dello Stato; Şevket Kazan, Ministro della Giustizia; Oğuzhan Asıltürk, Ministro degli Interni; Fehmi Adak, Ministro del Commercio; Korkut Özal, Ministro dell'Approvvigionamento, Agricoltura e Allevamento; Abdülkerim Doğru, Ministro dell'Industria e della Tecnologia.

23 Tra il 1975 e il 1980 si ebbero un governo di transizione guidato da Sadi Irmak, tre governi guidati da Demirel, (due dei quali con l'appoggio di Erbakan, Fronte nazionalista), e due governi di Ecevit.
} 


\section{Il colpo di stato del 1980}

L'esercito ha da sempre occupato un ruolo preminente nel sistema politico turco ${ }^{24} \mathrm{e}$ si è imposto in diverse occasioni come guardiano del kemalismo: nel 1960, nel $1971 \mathrm{e}$ infine nel 198025. Il 12 settembre 1980, mentre era in carica il governo Demirel, l'esercito guidato dal generale Kenan Evren ${ }^{26}$ prese il potere, legittimando questo atto con la legge dei servizi interni. Secondo i vertici militari gli organi statali avevano smesso di funzionare, su iniziativa militare fu sciolto il Parlamento, il governo fu deposto, furono sciolti i partiti e arrestati gli uomini politici che fino a quel momento avevano retto le sorti nazionali. I militari ritenevano loro dovere salvare la democrazia dall'operato dei politici e si capì subito che nella Turchia dei militari non vi era posto per i vecchi politici. Erbakan fu arrestato e processato insieme a Alparslan Türkeș ${ }^{27}$, con l'accusa di aver progettato un cambiamento nell'assetto repubblicano, da laico a confessionale. Successivamente le accuse caddero e i due furono scagionati, ma si dovrà attendere il 1983 per vedere i vecchi leader politici tornare sulla scena politica. Erbakan lo fece con un nuovo partito islamico il Refah Partisi, che alle elezioni ottenne il 4,5\% dei voti non riuscendo a entrare al governo. La nuova formazione partitica prese avvio dalla mobilitazione politica del movimento Millî Görüșche vedeva nel partito una possibilità di rappresentanza politica. Nei primi anni Novanta ci fu una recrudescenza dei rapporti tra gli islamisti e i laici e accanto al partito di Erbakan si formarono altri gruppi più radicali28. Il partito islamico attraversò una fase di crescita che durò dal 1983 fino al 1991, anni in cui alle diverse tornate elettorali si registravano continui

24 In Turchia l'esercito ha costruito la Repubblica attraverso la guerra di Liberazione Nazionale, questo gli conferisce un potere enorme, rendendolo un simbolo nazionale e, al contempo, uno strumento per preservare la nazione voluta da Atatürk e portata avanti dai suoi successori, primo fra tutti Ismet Inönü.

${ }_{25}$ IMTIAZ, Ali, «Refletions on the army and the Islamist in Turkey», in Pakistan Horizon, 51, 1/1998, p. 64.

${ }^{26}$ Ahmet Kenan Evren è nato nel 1917 vicino a Manisa. Dopo il colpo di Stato del 1982 divenne uno degli uomini più potenti della Turchia. Lasciato l'esercito fu eletto Presidente della Repubblica, detenendo il potere fino al 1989.

27 Alparslan Türkeș, nato a Cipro nel 1917, durante la Seconda guerra mondiale era un fervente panturchista, fu uno dei principali organizzatori del colpo di Stato del 1960. Nello stesso anno divenne addetto militare in India. Tornò in patria nel 1965 e poco dopo diventò il leader dell'ultranazionalista Partito d'Azione Nazionalista. Collegato al Partito il movimento dei Lupi Grigi, movimento nazionalista accusato di vari attentati, il più famoso quello compiuto ai danni di Giovanni Paolo II. Dopo il colpo di stato del 1980 Alparslan fu allontanato dalla politica, rientrò in scena nel 1987. Morì dieci anni dopo.

${ }_{28}^{28}$ Tra essi il più famoso, anche tra i turchi emigrati in Europa, era il gruppo della Voce nera, di Hoca Cemalettin Kaplan, che viveva in Germania e teorizzava uno Stato islamico, alla stregua dell'ayatollah Khomeyni in Iran. 
successi $^{29}$. Il partito conquistò consensi anche nei bassi ranghi dell'esercito e nel 1987 molti cadetti che frequentavano l'accademia militare furono espulsi perché accusati di attività islamiste; lo stesso accadde nel 1994 ad alcune decine di membri della marina militare sospettati dello stesso reato.

\section{Anni Novanta, nuova vita per l'Islam politico}

Le elezioni politiche del 1991 decretarono il ritorno al potere di Demirel ma sancirono anche la rinascita del partito di Erbakan, che riuscì a ottenere il 17\% dei consensi, anche se si deve considerare l'alleanza con gli ultranazionalisti di Türkeș. Le elezioni del 1994 rappresentarono un altro banco di prova importante e rivelarono l'importanza che stava assumendo il Refah all'interno del panorama politico turco. Le città chiave della Turchia, Istanbul e Ankara, finirono in mano al partito islamico, che raddoppiò i consensi rispetto alle precedenti elezioni. Il successo elettorale derivò essenzialmente dalla campagna portata avanti creando uno stretto contatto con la gente, soprattutto nelle periferie urbane delle grandi città, dove si registrò il consenso maggiore. Importante fu il contributo delle donne islamiste ${ }^{30}$ durante la campagna elettorale ${ }^{31}$. Il Refah si propose di risolvere la questione kurda offrendo una piattaforma di unità tra turchi e kurdi - senza escludere le altre minoranze - basata sull'Islam. Le linee guida del partito indicavano un riconoscimento dell'identità kurda e proponevano la libertà di espressione culturale, ma i mass media attaccarono duramente il partito, accusato di essere dalla parte dei kurdi contro l'identità nazionale turca. In economia veniva proposto il cosiddetto giusto ordine economico, basato sull'equità economica, dettata dall'Islam. Allo stesso tempo le linee guida si esprimevano in favore delle privatizzazioni ${ }^{2}$ e Erbakan prometteva una rigenerazione morale della nazione, la lotta alla corruzione, aiuti alle imprese private, libertà di espressione per tutti: erano questi i punti che avrebbero fatto della Turchia una nazione libera dall'Occidente 33 .

\footnotetext{
29 Alle elezioni comunali del 1984 il Refah ottenne il governo di 17 comuni e due province, cinque anni dopo i comuni controllati erano 100.

30 Per approfondimenti vedere: ARAT, Yeșim, «On Gender and Citizenship in Turkey», in Middle East Report, 1/1996, pp. 28-31.

${ }^{31}$ DEVRIM-BOUVARD, Nukte, «Turkish Women and the Welfare Party. An interview with Şirin Tekeli», in Middle East Report, april-june 1996, p. 28.

$3^{2}$ GÜLALP, Haldun, «Islamist Party Poised for National Power in Turkey», in Middle East Report, 194-195/1995, p. 56.

33 FEROZ, Hamed, "Politics and Islam in Modern Turkey», in Middle Eastern Studies, 27, 1/1991, p. 16.
} 


\section{Elezioni del 1995 e governo Erbakan}

La fase più importante delle vicende legate all'islam politico e alla figura politica e personale di Erbakan inizia alla fine del 1995, quando le elezioni confermarono, rafforzandola, la tendenza che si era già registrata nella precedente tornata elettorale. Il Refah diventò il primo partito del paese, con il 21, 32\% dei voti e questo risultato segnò uno spartiacque nella storia della Repubblica turca. Ebbe così il periodo d'oro dell'Islam politico. Il Partito della Madre Patria arrivò secondo e il Partito della Giusta Via, terzo 34 . Erbakan venne incaricato della formazione del governo ma quasi subito dovette rinunciare poiché non riuscì a ottenere l'appoggio di nessuno dei partiti all'opposizione ${ }^{35}$. I due partiti, capeggiati da Mesut Yilmaz ${ }^{36}$ e da Tansu Çiller37, cercarono di formare un governo di coalizione ma, a causa delle divergenze personali tra i due, non vi riuscirono. Vista la situazione di crisi politica il presidente Demirel decise di affidare l’incarico di formare un governo al vincitore delle elezioni, Erbakan, che si mise subito al lavoro per formare il suo primo governo. Questa volta riuscì nell'intento, perché a sorpresa Tansu Çiller, in passato sua acerrima oppositrice, decise di affiancarlo e di collborare con lui ${ }^{3}$. Una strana coalizione che vedeva fianco a fianco Islamisti e laici. Tansu Çiller ricoprirà il ruolo di vice primo ministro e ministro degli esteri, il suo partito avrà i dicasteri più importanti quali, industria, difesa e interni. Dopo aver ottenuto la fiducia del Parlamento39 iniziò l'avventura di questo peculiare governo, che potè contare su 550 seggi. Nacque così la "Grande Unità" tra gli islamisti di Erbakan e gli ultra laici della Çiller, che dovette affrontare una serie di emergenze,

34 FERRARI, Antonio, «Turchia patto laico contro gli islamici», in Corriere della Sera, 27 dicembre 1995, p. 7.

35 FERRARI, Antonio «Turchia, Erbakan rinuncia», in Corriere della Sera, 20 gennaio 1996, p. 9.

${ }_{36}$ Mesut Yılmaz è nato nel 1947 a Istanbul; nel 1971 si è laureato in scienze politiche all'Università di Ankara, proseguendo poi gli studi a Colonia. Nel 1983 è stato tra i fondatori del Partito della Madrepatria; è stato nominato dapprima Ministro degli Esteri e poi, per due volte, nel 1991 e nel 1996, Primo ministro.

37 Tansu Çiller è nato a Istanbul nel 1946; dopo aver ricevuto un'istruzione di tipo occidentale si dedica alla carriera accademica. È entrata in politica all'inizio degli anni Novanta, diventando in breve tempo leader del Partito della Giusta Via e subito dopo Primo ministro (1993). È stata protagonista della scena politica fino al 1996; a partire da questa data si aprì un lungo periodo di declino che culminò con il ritiro, nel 2002.

${ }^{8}$ Varie fonti ventilavano l'ipotesi che i due leader avessero raggiunto l'accordo perché Erbakan promise di votare contro l'inchiesta che coinvolgeva la Çiller per presunti fondi neri; in cambio la Çiller lo sostenne e votò assieme ai compagni di partito la fiducia in Parlamento.

39 BOHLEN, Celestine, «Islamic-Secular Coalition Cabinet Is Approved in Turkey», in New York Times, 9 July 1996. 
economia, sicurezza e crisi curda ${ }^{40}$. L'ascesa al potere del partito islamico creò uno shock molto importante nel sistema politico turco, da decenni guidato secondo i principi kemalisti attraverso governi non confessionali ${ }^{41}$. I militari lo guardarono con sospetto e dichiararono che avrebbero controllato da vicino le azioni del governo: in pratica il premier divenne un sorvegliato speciale ${ }^{42}$. Nei primi mesi il governo si impegnò nel dare solidità all'esecutivo e furono abbandonate sia le istanze nazionalistiche, come per esempio le richieste di nazionalizzazione delle grandi imprese, sia la retorica islamica43. Il programma di governo prevedeva una serie di misure miranti all'equità sociale, che includevano giustizia, sviluppo economico, lotta alla corruzione, protezione della proprietà, rafforzamento del rapporto Stato-nazione e fine dell'influenza occidentale sulla Turchia44. Dopo circa sei mesi la coalizione sembrava aver raggiunto una certa stabilità e i due leader avevano tutte le ragioni per fare in modo che questa unità continuasse: la Çiller viste le accuse di corruzione a suo carico non poteva permettersi di interrompere l'idillio e Erbakan, che vedeva crescere il suo consenso, non poteva permettersi di fallire. L'unico neo erano i rapporti tra governo ed esercito. La Çiller spesso era riuscita a mediare tra i militari e il Refah ma nel 1997 la situazione improvvisamente precipitò. Ci furono una serie di iniziative da entrambe le parti che non fecero che deteriorare ulteriormente i rapporti. Le denunce dei militari avevano un solo obiettivo: convincere la Çiller a abbandonare il governo e costringere così Erbakan alle dimissioni ${ }^{45}$. Il governo rimane in piedi ancora per qualche mese, perché Erbakan dichiarò di voler seguire i "consigli" dei militari e di non voler far diventare la Turchia un paese con un governo confessionale. La situazione politica era molto instabile: Erbakan a capo di un governo di coalizione con un partito scomodo come il Partito della Giusta Via; la Çiller, era pressata dall'esercito affinché abbandonasse l'esecutivo. Il governo per ovviare a questa situazione annunciò elezioni anticipate, giustificando l'annuncio con l'impellente necessità di nuovi successi

40 FERRARI, Antonio «Erbakan salvato dai voti della Çiller. Via al governo», in Corriere della Sera, 9 luglio 1996, p. 8.

${ }^{41}$ AYDITAȘBAS,, Aslı, «The Malaise of Turkish Democracy», in Middle East Report, 209/1998, p. 33 .

${ }^{2}$ FERRARI, Antonio, «Turchia: il premier sorvegliato speciale», in Corriere della Sera, 30 giugno 1996, p. 7.

43 Per quanto riguarda la politica estera, Erbakan si dedicò alla costituzione di un gruppo economico denominato D8, che costituiva secondo il premier un alternativa economica all'Europa.

44 YAVUZ, Hakan M., "Political Islam and the Welfare (Refah) Party in Turkey», in Comparative Politics, 30, 1/1997, p. 73.

45 FERRARI, Antonio, «I militari processano Erbakan», in Corriere della Sera, 1 marzo 1997, p. 12. 
economici. In realtà sembrava che il governo fosse oramai incapace di guidare il paese 46 .

L'epilogo appariva quasi scontato: Erbakan a metà giugno decise di rassegnare le dimissioni, ponendo fine all'anno più importante della storia dell'Islam politico in Turchia e chiudendo l'esperienza di governo. Dopo aver cercato di resistere alle pressioni dei militari, di una parte dei mass media e della società civile, che non accettava la prospettiva di uno stato confessionale, fu costretto a cedere. Anche l'ultima delle sue proposte, cioè di cedere il comando alla Çiller, trovò l'opposizione del presidente Demirel, che non concesse il rimpasto di governo. L'ultimo atto fu quello delle dimissioni per cercare di dare un nuovo governo alla Turchia, che continuava a attraversare un periodo di profonda crisi economica, sociale, e politica. L'esercito riuscì così a portare a termine quello che è conosciuto come il primo colpo di stato postmoderno.

\section{Processo contro il Refah}

Nel maggio del 1997 il partito Refah fu accusato di appoggiare il fondamentalismo islamico e di voler sovvertire l'ordinamento laico della Turchia. Si trattò di un duro attacco nei confronti del leader Erbakan, e di alcuni dei suoi esponenti di spicco come Recep Tayyp Erdoğan 47 che furono accusati di incitamento all'odio religioso e furono arrestati, processati e condannati.

Nel gennaio 1998 la Corte Costituzionale decise di far dichiarare fuori legge il partito ${ }^{48}$ e di vietare a Erbakan di praticare attività politica per cinque anni49. La chiusura del Refah e la messa al bando dei suoi dirigenti, chiuse una campagna ispirata dai militari che non hanno mai gradito la presenza degli islamisti nel contesto politico nazionale. Gli islamisti corsero ai ripari e Recep Tayyp Erdoğan e Abdüllah Gul5o, che

46 FERRARI, Antonio, «Turchia, voto anticipato Erbakan messo all'angolo», in Corriere della Sera, 2 giugno 1997, p. 12.

47 Recep Tayyip Erdoğan è nato a Istanbul nel 1954. Ha intrapreso l'attività politica alla fine degli anni Settanta diventando leader del Partito per la Giustizia e lo Sviluppo. Il suo partito vinse le elezioni legislative del 3 novembre 2002, è primo ministro dal 14 marzo 2003. Figura di spicco del disciolto Partito del Benessere, di ispirazione islamico-conservatrice, è divenuto una figura politica di rilevanza nazionale come sindaco di Istanbul.

48 Per approfondimenti vedere: KOGACIOĞLU, Dicle, «Progress, Unity, and Democracy: Dissolving Political Parties in Turkey», in Law \& Society Review , 38, 3/2004, pp. 433-462.

49 RAMPOLDI, Guido, «Turchia, fuorilegge gli Islamici», in la Repubblica, 17 gennaio 1998, p. 15 .

50 Abdullah Gül (1950), economista e professore universitario, ha studiato scienze economiche alla Facoltà di Economia dell'Università di Istanbul. È stato eletto in Parlamento nel 1991 come deputato del collegio di Kayseri tra le file del Partito del Benessere (Refah Partisi). A seguito 
all'interno del Refah rappresentavano l'ala più progressista, fondarono il Partito della Virtù (Fazilet Partisi) ${ }^{51}$, ma anche questa formazione partitica fu posta fuori legge. I progressisti, usciti sconfitti anche in un conflitto interno, fondarono allora il Partito della Giustizia e dello Sviluppo (Adalet ve Kalkinma Partisi).

Intanto Erbakan52, dopo aver invitato gli aderenti al partito a mantenere la calma, non si diede per sconfitto, si rivolse alla Commissione per i Diritti Umani del Consiglio d'Europa e continuò il suo impegno politico fondando il Partito della Felicità (Saadet Partisi).

La storia recente vide l'ascesa del Partito della Giustizia e dello Sviluppo, che alle elezioni del 2002 sbaragliò tutti i partiti avversari53. I turchi votarono Erdoğan perché lo ritenevano capace di eliminare la povertà54 e la corruzione e non perché desideravano uno stato islamico55. La percezione dell'uomo comune era cambiata, era finita l'era d'oro dell'Islam politico, almeno di quello rappresentato da Necmettin Erbakan.

Anche se Erdoğan è considerato il successore politico di Erbakan ${ }^{6}$, quest'ultimo negli anni non mancò di criticare l'operato del suo delfino57, accusando il governo guidato da Erdoğan di essersi avvicinato politicamente all'Europa e a Israele58. Erdoğan, almeno nei primi anni di governo, si era schierato inequivocabilmente per l'ingresso della Turchia in Europa. Questa posizione era giustificata da ragioni

della dissoluzione del Partito è stato tra i fondatori del Partito della Virtù (Fazilet Partisi). Allo scioglimento di questo, nel 2001, ha aderito al Partito per la Giustizia e lo Sviluppo (Adalet ve Kalkınma Partisi). La sua carriera politica è stata in continua ascesa: dopo essere stato deputato in varie legislature, è stato nominato Ministro degli Esteri nel marzo del 2003, Primo Ministro dal 18 novembre 2002 all'11 marzo 2003 ed è attualmente Presidente della Repubblica di Turchia.

${ }^{51}$ Questo partito fu disciolto nel 2001: al suo interno si accese una disputa tra l'ala conservatrice e l'ala progressista che voleva trasformare il partito in un movimento di centrodestra che non faceva più ricorso alla retorica religiosa.

$5^{2}$ DUMAN, Ismail, «Divisions in Erbakan's movement», in Islamic Awakening, 1, 8/2013, p. 14. 53 Per approfondimenti vedere: UGUR, Ikinci, «The Walfare Party's Municipal Track Record: Evaluating Islamist Municipal Activism in Turkey», in Middle East Journal, 53, 1/1999, pp. 7594.

54 Per approfondimenti vedere: SEBNEM, Gumuscu, DENIZ Sert, «The Power of the Devout Bourgeoisie: The Case of the Justice and Development Party in Turkey», in Middle Eastern Studies, 45, 6/2009, pp. 953-968.

55 Questa teoria è dimostrata dai risultati elettorali che vede gli islamisti del Partito della Felicità sconfitti anche nella loro roccaforte storica, Konya.

${ }^{56}$ SHANKLAND, David, «Islam and Politics in Turkey: the 2007 Presidential Elections and Beyond», in International Affairs 83, 2/2007, p. 361.

57 Anche se entrambi sono considerati islamisti, la loro politica, specialmente quella estera è completamente differente, per approfondimenti su quest'aspetto vedere: BRENT, E. Sasley, «Turkish Leaders and Foreign Policy Decision Making: Lobbying for European Union Membership», in Middle Eastern Studies 48, 4/2012, pp. 553-566.

${ }_{58}$ BOZKURT, Abdullah, «Erbakan criticizes Israel accuses Erdogan of being part of Jewish cospiracy», in Today's Zaman, 12 juny 2006, p. 1. 
economiche, il nuovo leader turco in contrapposizione con il suo predecessore, si dichiarò favorevole al capitalismo, associando una rapida ripresa economica con una altrettanto veloce riduzione dell’inflazione. Tutto ciò non escludeva però i rapporti con gli altri paesi musulmani: nel primo decennio degli anni Duemila ci fu un incremento degli investimenti economici in paesi come l'Arabia Saudita59.

\section{Conclusioni}

Erbakan, con la sua decennale carriera politica, è stato uno dei più importanti uomini politici della Turchia. La sua importanza è espressa da diversi fattori che hanno concorso a fare di lui uno degli uomini più importanti della storia recente del Paese. Egli ha lasciato la sua impronta nella società civile turca in diversi modi, il più importante è l'introduzione dell'Islam politico in una nazione che da settanta anni si reggeva sul principio inviolabile e imprescindibile della laicità di Stato. Questa sua azione ha creato una frattura nella società e nel contempo una nuova consapevolezza per quanti erano sottoposti al kemalismo senza condividerne pienamente tutti i principi. Con la sua instancabile azione propagandistica ha cambiato il volto della politica nazionale, che era spesso statica perché basata sui principi di Atatürk, che risultavano datati considerando i passi avanti fatti dalla Turchia negli ultimi cinquanta anni. Il fatto stesso di aver creato varie formazioni partitiche di ispirazione islamica in un paese che lo vietava è un fattore innovativo. Erbakan, con i suoi estremismi e la sua dialettica, non era il solito politico cui la Turchia era abituata. Scosse gli animi, sia di chi condivideva i suoi ideali, sia di chi lo avversava nell'arena politica o attraverso i canali di informazione.

Il fatto di essere stato il primo capo di governo appartenente a una formazione politica di chiara ispirazione islamica, dopo più settanta anni dalla rivoluzione kemalista, ha creato uno spartiacque storico che ha fatto riprendere vigore all'Islam politico. Erbakan è l'ideologo di questo pensiero politico che unisce elementi nazionalistici, religiosi e di identità. Per tutti questi motivi Necmettin Erbakan, per quanto si tratti di una figura controversa, merita di essere considerato il padre dell'islam politico e uno degli innovatori della politica turca. I suoi ideali si possono condividere o meno ma ciò non toglie valenza alla sua azione politica e alla sua vicenda personale. I diversi partiti che ha concorso a fondare hanno rappresentato in Turchia l’islam politico per eccellenza.

59 PUPCENOKS, Juris, «Democratic Islamization in Pakistan and Turkey: Lesson for the Post Arab Spring Muslim World», in Middle East Journal, 66, 2/2012, p. 285. 
La Turchia ha riscoperto l'islamismo: il percorso è durato circa trenta anni e ha avuto come protagonista Erbakan, che è stato abile a sfruttare diversi fattori. Uno di questi fu certamente il crescente inurbamento di persone che provenivano dalle provincie economicamente più arretrate; questo incontro con la realtà cittadina, provocava una frizione e le persone si rifugiavano in quello che gli era più familiare: non lo Stato, ma la religione. L'altro fattore fu la crisi economica, che generò in sempre più estese fasce di popolazione insofferenza nei confronti dei politici e delle loro soluzioni. 


\section{* L'autore}

Emanuela Locci è dottore di ricerca presso la Facoltà di Scienze Politiche dell'Università degli Studi di Cagliari. Il suo principale filone di ricerca riguarda la storia della massoneria nell'Impero Ottomano e nell'area del Mediterraneo, con particolare attenzione ai paesi musulmani. È autrice di diversi paper e di Il cammino di Hiram. La massoneria dall'Europa all'Impero Ottomano (Foggia, Bastogi Editrice Italiana, 2013).

URL: < http://www.studistorici.com/progett/autori/\#Locci >

\section{Per citare questo articolo:}

LOCCI, Emanuela, «L'Islam di Stato. La figura di Necmettin Erbakan nella Turchia contemporanea», Diacronie. Studi di Storia Contemporanea : Periferie. Cultura, economia, politica, 29/3/2014,

URL: < http://www.studistorici.com/2014/3/29/Locci_numero_17/ >

Diacronie Studi di Storia Contemporanea www.diacronie.it

Risorsa digitale indipendente a carattere storiografico. Uscita trimestrale. redazione.diacronie@hotmail.it

Comitato di redazione: Jacopo Bassi - Luca Bufarale - Elisa Grandi - Deborah Paci - Fausto Pietrancosta - Matteo Tomasoni - Luca Zuccolo

Diritti: gli articoli di Diacronie. Studi di Storia Contemporanea sono pubblicati sotto licenza Creative Commons 2.5. Possono essere riprodotti a patto di non modificarne i contenuti e di non usarli per fini commerciali. La citazione di estratti è comunque sempre autorizzata, nei limiti previsti dalla legge. 